\title{
Molecular Modeling Studies on Binding of Chlorotetracycline and Enoxacin to Human Serum Albumin
}

\author{
Taoying $\mathrm{Hu}^{1,}$, Ying $\mathrm{Liu}^{* 1,2, \mathrm{~b}}$ \\ ${ }^{1}$ College of Life and Environmental Science, Minzu University of China, Beijing 100081 China \\ ${ }^{2}$ Beijing Engineering Research Center of Food Environment and Public Health, Minzu University of \\ China, Beijing 100081 China \\ aemail: hty0945020@163.com, 'email: liuying4300@163.com \\ *Corresponding author. Tel.:+86 15810128921; Fax: +86 1068450641
}

Keywords: Molecular Modeling; Chlorotetracycline; Enoxacin; Human Serum Albumin

\begin{abstract}
The interactions of chlorotetracycline (CTC) and enoxacin (ENX) with human serum albumin (HSA) had been studied by molecular modeling method. Molecular docking revealed that both CTC and ENX bound to site I (subdomain IIA) of HSA through multiple interactions, such as hydrogen bonds, hydrophobic effect and polar force. Moreover, the systems' (CTC-HSA and ENX-HSA) Gibbs free energies $(\Delta G)$ were $-27.50 \mathrm{~kJ} \cdot \mathrm{mol}^{-1}$ and $-29.93 \mathrm{~kJ} \cdot \mathrm{mol}^{-1}$, respectively.
\end{abstract}

\section{Introduction}

Human serum albumin (HSA), the most abundant serum protein in blood plasma, plays a major role in the transport and deposition of many endogenous and exogenous drugs ligands in blood. A large variety of drugs bind with serum albumins and their effectiveness depends on how well they bind with the proteins. Due to the availability of hydrophobic pockets inside the protein network and the flexibility of the albumins to adapt its shape, serum albumin can increase the apparent solubility of hydrophobic drugs in plasma and modulates their delivery to cells in vivo and in vitro [1]. Therefore, it is very important to study the interaction of the ligands with HSA.

Chlorotetracycline, a tetracyclines antibiotic, is widely used for the treatment of primary and secondary infections in respiratory, digestive, urinary tracts and multisystem infection due to its broad-spectrum antibiotic activity. Enoxacin, a new fluoroquinolone human and veterinary antibiotic, which is widely used for the prevention and therapy of dermatological, respiratory and urinary diseases. Although the interactions of CTC and ENX with HSA have been studied by using multi-spectroscopic methods [2][3], but molecular modeling, which can offer a molecular level explanation with the ability to estimate the participation of specific chemical groups and their interactions in complex stabilization, has not previously been reported. The aspect hinders our proper and comprehensive understanding of the interactions between CTC/ENX and HSA.

In the present work, molecular modeling technique was employed to demonstrate the interactions of CTC and ENX with HSA. The possible interaction sites of CTC-HSA and ENX-HSA systems were investigated, and the relevant structure and energies of the binding sites were determined through protein ligand docking studies. Molecular modeling studies were carried out by using DockingServer.

\section{Molecular Modeling Study}

Docking calculations were performed with DockingServer on a HSA protein model. The MMFF94 force field was used for energy minimization of the ligand molecule. Gasteiger partial charges were added to the ligand atoms. Non-polar hydrogen atoms were merged, and rotatable bonds were defined.

According to paper [4], docking calculations and simulations were carried out on CTC and ENX with HSA protein model. 


\section{Results and Discussion}

Molecular Docking Study of the Interaction between CTC and HSA. HSA is a globular protein composed of three homologous $\alpha$-helical domains (I-III). Each domain contains two subdomains (A and B; Figure1 (a)). The principal regions of ligand binding sites of HSA are located in hydrophobic cavities in subdomains IIA and IIIA. Site I (subdomain IIA), which is known as the warfarin binding site, prefers to bind with large heterocyclic and negatively charged compounds, while site II (subdomain IIIA) is a suitable binding site for small aromatic carboxylic acids, and only one tryptophan residue (Trp-214) of HSA is in subdomain IIA [5].
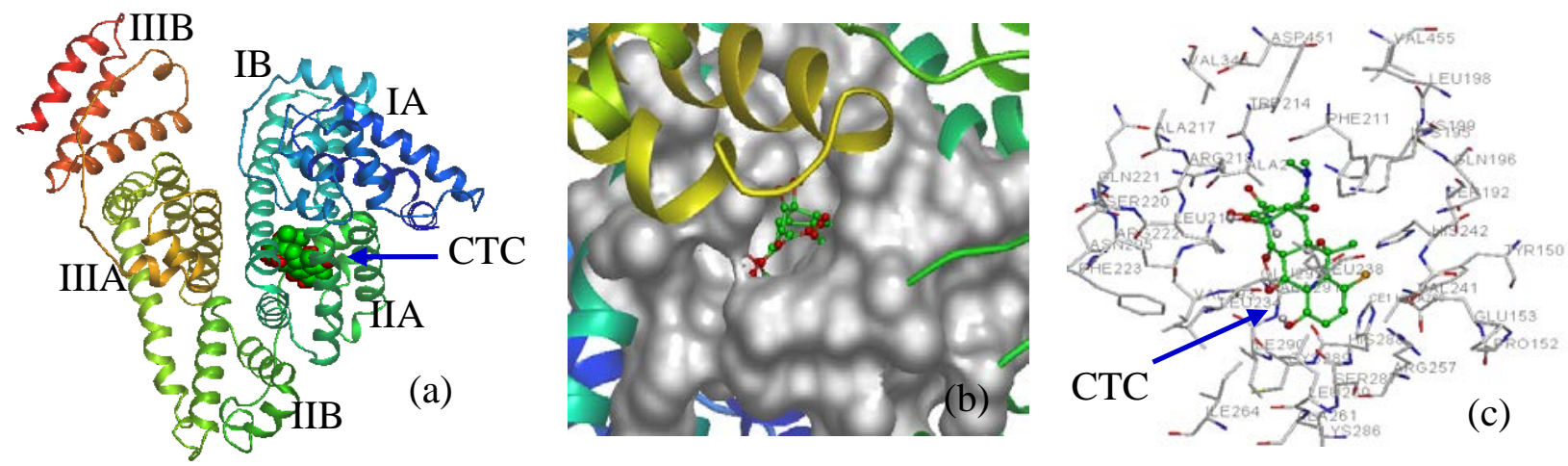

Fig.1. (a) Domain structure of HSA and the binding site of CTC on HSA. HSA is shown in cartoon and CTC is represented using spheres. (b) Enlarged binding mode between CTC and HSA. HSA is shown in cartoon, the interacting side chains of HSA are displayed in surface mode and CTC is represented using balls and sticks. (c) The interaction mode between CTC and HSA. The residues of HSA are represented using cylinders and CTC structure is represented using balls and sticks.

Table 1. Atoms involved in the hydrogen bonds, polar and hydrophobic interaction of CTC with HSA and the distance between them, analyzed from the molecular docking results

\begin{tabular}{lccl}
\hline \multicolumn{1}{c}{ Protein atom (HSA) } & Ligand atom (CTC) & Distance $(\AA)$ & Driving force \\
\hline Arg218(CZ,NE,NH2) & O3 & 3.05 & hydrogen bonds \\
Arg222(CZ,NH1,NH2) & O14 & 2.68 & hydrogen bonds \\
Arg222(NH2) & O3 & 3.01 & hydrogen bonds \\
Ala291(O) & N1 & 2.49 & hydrogen bonds \\
Leu219(CD1) & C18 & 3.80 & hydrophobic \\
Leu238(CD1,CD2,CG) & C19 & 3.07 & hydrophobic \\
Leu238(CD1,CD2) & C26 & 3.61 & hydrophobic \\
Leu238(CD1) & C25 & 3.82 & hydrophobic \\
Leu238(CD2) & C20 & 3.25 & hydrophobic \\
Leu238(CD2) & C18 & 3.31 & hydrophobic \\
His242(CE1) & C22 & 2.93 & hydrophobic \\
Ala291(CB) & C24 & 3.58 & hydrophobic \\
Ala291(CB) & C32 & 3.23 & hydrophobic \\
Ala291(CB) & C31 & 3.45 & hydrophobic \\
Lys199(NZ) & N11 & 2.59 & polar \\
Lys199(NZ) & O23 & 3.83 & polar \\
Arg218(CB,CG) & O15 & 3.00 & polar \\
Arg218(CD,CG,CZ,NE,NH2) & O14 & 2.85 & polar \\
Arg222(CZ,NH1,NH2) & N1 & 3.15 & polar \\
Arg222(NH1) & O16 & 3.05 & polar \\
\hline
\end{tabular}


The crystal structure of HSA was taken from the Protein Data Bank (entry PDB code 3LU7). The best energy ranked result is shown in Figure1. From Figure1, it can be seen that CTC bound within the subdomain IIA of the protein, and the inside wall of the pocket of subdomain IIA is formed by hydrophobic side chains, whereas the entrance to the pocket is surrounded by positively charged residues consisting of Trp214, Lys199, Lys195, Arg218, Arg257 and His242. Thus we can conclude that CTC was able to fit well within the subdomain IIA hydrophobic cavity (see Figure1 (b)). The docking result showed the existence of hydrogen bonds, hydrophobic and polar interactions between CTC and HSA (Table 1), for example, N1 and O16 of CTC interacted with Arg222 through polar force. Furthermore, the distances were measured between the protons of CTC and the adjacent residues around the binding site. Active site residues within $5 \AA$ to CTC included Ala 291, Arg222, Tyr150, Leu238, Leu219 and Ile290. The calculated binding Gibbs free energy $(\Delta G)$ was $-27.50 \mathrm{~kJ} \cdot \mathrm{mol}^{-1}$.

Molecular Docking Study of the Interaction between ENX and HSA. The crystal structure of HSA was taken from the Protein Data Bank (entry PDB code 3LU7). The best energy ranked result is presented in Figure2. The docking result showed that ENX was located within the binding pocket of subdomain IIA of the protein, and ENX was adjacent to hydrophobic residues Leu219, Leu238, Leu260, Ile290, Ile264 and Ala291 of subdomain IIA of HSA. On the other hand, there were hydrogen bonds between O10 of ENX and the residues Lys199 of HSA; N18 and Ser287 (Table 2). The hydrogen bonding between ENX and the side chains of amino acid residues in HSA could impact the formation of the $\mathrm{C}=\mathrm{O}$...HO-C intramolecular hydrogen bond, resulted in decrease in the polarity of these groups, which leaded to an increase in hydrophobicity and decrease in hydrophilicity. Moreover, the docking result showed the existence of polar interaction and halogen bond between ENX and HSA, for example, F17 of ENX interacted with Tyr150 through halogen bond. The calculated binding Gibbs free energy $(\Delta G)$ was $-29.93 \mathrm{~kJ} \cdot \mathrm{mol}^{-1}$.
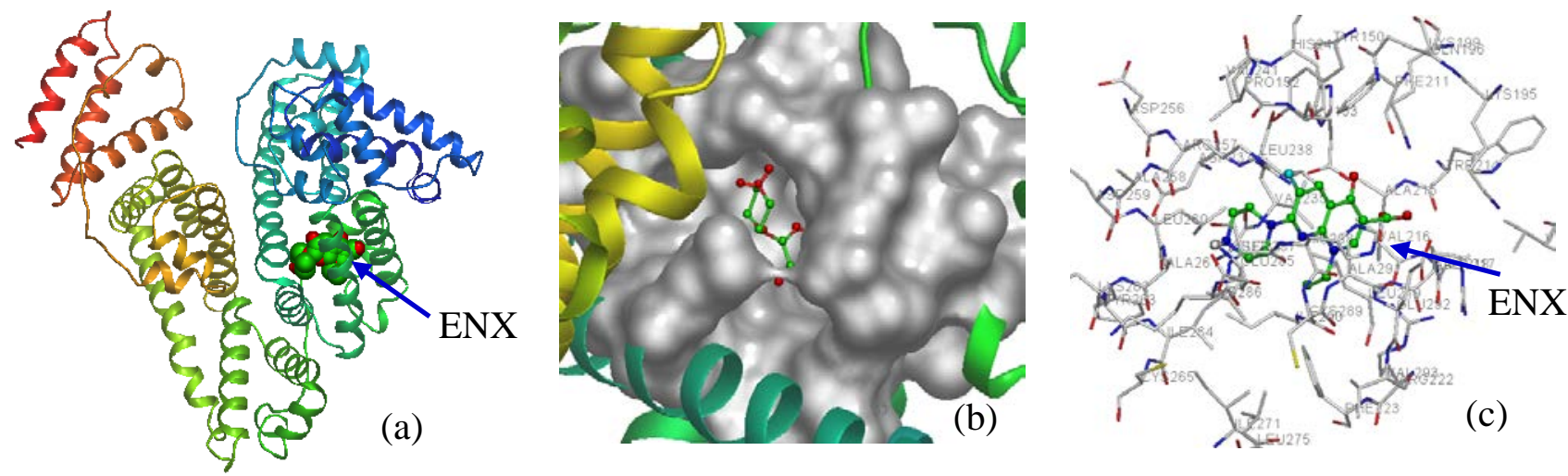

Fig.2. (a) Structure of HSA and the binding site of ENX on HSA. HSA is shown in cartoon and ENX is represented using spheres. (b) Enlarged binding mode between ENX and HSA. HSA is shown in cartoon, the interacting side chains of HSA are displayed in surface mode and ENX is represented using balls and sticks. (c) The interaction mode between ENX and HSA. The residues of HSA are represented using cylinders and ENX structure is represented using balls and sticks.

Table 2. Atoms involved in the hydrogen bonds, polar, hydrophobic interactions and halogen-bond of ENX with HSA and the distance between them, analyzed from the molecular docking results

\begin{tabular}{lccl}
\hline Protein atom (HSA) & Ligand atom $(\mathrm{ENX})$ & Distance $(\AA)$ & Driving force \\
\hline Lys199(NZ) & O10 & 3.42 & hydrogen bonds \\
Ser287(CB,O) & N18 & 3.27 & hydrogen bonds \\
Leu219(CD2) & C9 & 3.54 & hydrophobic \\
Leu219(CD2) & C12 & 3.68 & hydrophobic \\
Leu238(CD1) & C16 & 3.51 & hydrophobic \\
Leu238(CD2) & C4 & 3.61 & hydrophobic \\
\hline
\end{tabular}




\begin{tabular}{lcll}
\hline Leu260(CB,CD2,CG) & C20 & 3.14 & hydrophobic \\
Ile264(CD1) & C22 & 3.26 & hydrophobic \\
Ile290(CB,CG2) & C23 & 3.28 & hydrophobic \\
Ile290(CG2) & C12 & 3.74 & hydrophobic \\
Ala291(CB) & C7 & 3.71 & hydrophobic \\
Arg218(CB,CG) & O1 & 3.34 & polar \\
His242(NE2) & O10 & 3.31 & polar \\
Ser287(OG) & N21 & 3.84 & polar \\
Tyr150(OH) & F17 & 3.04 & halogen-bond \\
\hline
\end{tabular}

\section{Conclusion}

In this paper, we simulated the bindings of CTC and ENX with HSA by molecular modeling technique. Molecular docking result showed the details in the modeled CTC-HSA and ENX-HSA structures. CTC can interact with HSA in site I (subdomain IIA) through hydrogen bonds, hydrophobic and polar interactions, while ENX was located in subdomain IIA through hydrogen bonds, hydrophobic, polar interactions and halogen-bond. The study is helpful for understanding its effect on protein function during its transportation and distribution in blood.

\section{Acknowledgement}

Here the authors thank the National Science Foundation of China (21177163), 111Project B08044, Beijing Engineering Research Center of Food Environment and Public Health, Minzu University of China (10301-01404026), First-class University First Class Academic Program of Minzu University of China (YLDX01013), Graduate Student Scientific Research Innovation Project of Minzu University of China (K2014042).

\section{References}

[1] Mei Yan, Xin Chen, Shuting Sun, et al. Study on binding mechanism of meso-tetra-(3,5-dibromo-4-hydroxyphenyl) porphyrin with protein by fluorescence method [J]. Spectroscopy and Spectral Analysis, 200828 (6) 1322-1326.

[2] Yongnian Ni, Qiuhong Liu, Serge Kokot. Spectrophotometric study of the interaction between chlorotetracycline and bovine serum albumin using Eosin $\mathrm{Y}$ as site marker with the aid of chemometrics [J]. Spectrochimica Acta Part A: Molecular and Biomolecular Spectroscopy, 201178 (1) $443-448$.

[3] Yongming Liu, Guizhi Li. Interaction of enoxacin with albumins by fluorescence quenching analysis [J]. Chinese Journal of Applied Chemistry, 200421 (6) 621-624.

[4] Chengyu Dong, Ningning Lu, Ying Liu. Binding of methacycline to human serum albumin at subdomain IIA using multispectroscopic and molecular modeling methods [J]. Luminescence, 2013 28 (6) 933-941.

[5] Ulrich Kragh-Hansen, Victor Tuan Giam Chuang, Masaki otagiri. Practical aspects of the ligand-binding and enzymatic properties of human serum albumin [J]. Biological and Pharmaceutical Bulletin, 200225 (6) 695-704. 